\title{
Organizing Pneumonia as the First Clinical Manifestation of Early Stage Rheumatoid Arthritis Determined by Hand Joints Synovitis Using Magnetic Resonance Imaging
}

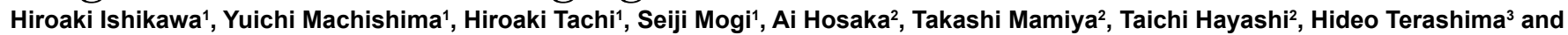
Shinji Teramoto ${ }^{*}$

${ }^{1}$ Department of Internal Medicine, Hitachi, Ltd. Hitachinaka General Hospital, Japan

${ }^{2}$ Department of Internal Medicine, Hitachinaka Medical Education and Research Center, The University of Tsukuba, Japan

${ }^{3}$ Department of Surgery, Hitachinaka Medical Education and Research Center, The University of Tsukuba, Japan

\begin{abstract}
We report the case of a 50-year-old man who presented with organizing pneumonia (OP) as the first manifestation of rheumatoid arthritis (RA). He experienced repeated episodes of pneumonia, which did not respond to several antibiotics. The lymphocyte dominant cell increase in the bronchoalveolar lavage fluid on chest computerized tomography suggested OP. Although he did not present with articular symptoms, magnetic resonance imaging (MRI) revealed synovitis of the hand joints without joint erosion, suggesting that this was a case of early stage RA. The MRI may be a useful diagnostic tool in asymptomatic patients with early stage RA.
\end{abstract}

Keywords: Organizing pneumonia; Rheumatoid arthritis; Joints synovitis; Magnetic resonance imaging

\section{Introduction}

There has been increasing recognition of the importance of respiratory disease in patients with rheumatoid arthritis (RA). Interstitial lung disease (ILD) is the only complication of RA that is increasing in prevalence and accounts for approximately $6 \%$ of all RA deaths [1]. Organizing pneumonia (OP) is a type of RA-associated interstitial lung diseases (RA-ILD). However, the joint symptoms of RA are usually dominant over the lung alterations in the patients with RA-ILD. Thus, ILD including OP without joint involvement may not be recognized as a sign of RA. Here, we report a case of early stage RA with OP as its first manifestation and accompanied by joint synovitis, which was identified on an MRI scan; MRI has recently become the established method for identification of the insidious joint involvement in RA [2,3].

\section{Case Report}

A 50-year-old man with symptoms of sore throat, dry cough, and progressive dyspnea towards the end of March 2011, was treated repeatedly with different antibiotics, including levofloxacin, cefditoren pivoxil, minocycline hydrochloride, and ceftriaxone sodium hydrate; however, his symptoms did not resolve with these drugs. Therefore, he was transferred to our hospital. Although his chest radiographs indicated chronic pulmonary infiltration, his general condition appeared to be good. He had no symptoms of joint involvement, and had never experienced morning stiffness of the hands. His laboratory results upon admission were as follows: white blood cell count, 11,200 cells/ $\mu \mathrm{L}$; C-reactive protein (CRP), $4.59 \mathrm{mg} / \mathrm{mL}$; Mycoplasma IgM antibodies, (-) ; Legionella urinary antigen test (Binax NOW, Portland, ME,USA) (-); QuantiFERON-TB Gold (-); KL-6, 236 U/mL; SP-D, 218 $\mathrm{ng} / \mathrm{mL}$. Cultures of blood, sputum, and urine performed on several occasions, were all negative. His chest radiograph showed alveolar exudates with ground-glass opacities in the right lung, accompanied by pleuritis (Figure 1). A high-resolution computed tomography scan showed parenchymal exudates with lung volume loss, suggesting OP of the right lung (Figure 2). An analysis of bronchoalveolar lavage (BAL) fluid revealed lymphocyte dominant cell accumulation in his lungs. The ratio of CD4/CD8 was 4.03. The radiographic examination and BAL analysis were indicative of OP. We initiated treatment with administration of $30 \mathrm{mg}$ of prednisone. The radiographic abnormality was gradually resolved. Because the lung abnormality indicated OP, we took a brief past history and tested for antibodies of possible collagen

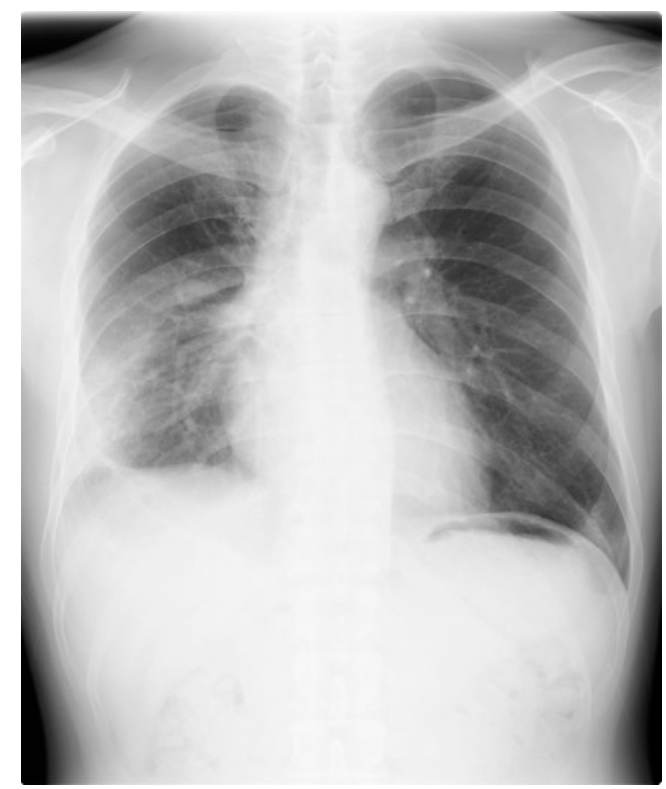

Figure 1: Chest radiograph on admission showed alveolar exudates with ground-glass opacities in the right lung, accompanied by pleuritis.

*Corresponding author: Shinji Teramoto, Hitachinaka Education and Research Center, The University of Tsukuba, Ishikawa-cho 20-1, Hitachinakashi, 312-0057 Ibaraki Japan, Tel: 81-29-354-5926; Fax: 81-29-354-5111; E-mail: shinjit-tky@umin.ac.jp

Received January 07, 2014; Accepted March 25, 2014; Published April 30, 2014

Citation: Ishikawa H, Machishima Y, Tachi H, Mogi S, Hosaka A, et al. (2014) Organizing Pneumonia as the First Clinical Manifestation of Early Stage Rheumatoid Arthritis Determined by Hand Joints Synovitis Using Magnetic Resonance Imaging Gen Med (Los Angel) 2: 138. doi: 10.4172/2327-5146.1000138

Copyright: (C) 2014 Ishikawa $\mathrm{H}$, et al. This is an open-access article distributed under the terms of the Creative Commons Attribution License, which permits unrestricted use, distribution, and reproduction in any medium, provided the original author and source are credited. 